\title{
Associations between schizophrenia genetic risk, anxiety disorders and manic/hypomanic episode in a longitudinal population cohort study
}

Alexander Richards, John Horwood, Joseph Boden, Martin Kennedy, Ruth Sellers, Lucy Riglin, Sumit Mistry, Hannah Jones, Daniel J. Smith, Stanley Zammit, Michael Owen, Michael C. O'Donovan and Gordon T. Harold

\section{Background}

Studies involving clinically recruited samples show that genetic liability to schizophrenia overlaps with that for several psychiatric disorders including bipolar disorder, major depression and, in a population study, anxiety disorder and negative symptoms in adolescence.

\section{Aims \\ We examined whether, at a population level, association between schizophrenia liability and anxiety disorders continues into adulthood, for specific anxiety disorders and as a group. We explored in an epidemiologically based cohort the nature of adult psychopathology sharing liability to schizophrenia.}

\section{Method}

Schizophrenia polygenic risk scores (PRSs) were calculated for 590 European-descent individuals from the Christchurch Health and Development Study. Logistic regression was used to examine associations between schizophrenia PRS and four anxiety disorders (social phobia, specific phobia, panic disorder and generalised anxiety disorder), schizophrenia/schizophreniform disorder, manic/hypomanic episode, alcohol dependence, major depression, and - using linear regression - total number of anxiety disorders. A novel population-level association with hypomania was tested in a UK birth cohort (Avon Longitudinal Study of Parents and Children).

\section{Results}

Schizophrenia PRS was associated with total number of anxiety disorders and with generalised anxiety disorder and panic disorder. We show a novel population-level association between schizophrenia PRS and manic/hypomanic episode.

\section{Conclusions}

The relationship between schizophrenia liability and anxiety disorders is not restricted to psychopathology in adolescence but is present in adulthood and specifically linked to generalised anxiety disorder and panic disorder. We suggest that the association between schizophrenia liability and hypomanic/manic episodes found in clinical samples may not be due to bias.

\section{Declarations of interest}

None.

\section{Keywords}

Schizophrenia; anxiety; polygenic risk score; CHDS; ALSPAC.

\section{Copyright and usage}

(c) The Royal College of Psychiatrists 2018. This is an Open Access article, distributed under the terms of the Creative Commons Attribution licence (http://creativecommons.org/licenses/by/ 4.0/), which permits unrestricted re-use, distribution, and reproduction in any medium, provided the original work is properly cited.
Schizophrenia is a debilitating, heritable disorder affecting between $0.5-1 \%$ of the population worldwide. ${ }^{1}$ Genetic factors contribute between $65-80 \%$ of the variance in risk and, of this, about a third to a half of the liability is distributed among many common risk alleles of small effect. ${ }^{2-4}$ At the individual level, the risk attributable to these alleles can be indexed by calculating polygenic risk scores (PRSs). Genetic studies of clinically ascertained cohorts have shown that schizophrenia PRS correlates with schizophreniaaffected status ${ }^{3,5}$ and also with other psychiatrically relevant traits, including bipolar disorder, ${ }^{3}$ major depression ${ }^{6}$ and poorer cognitive function. ${ }^{7,8}$ Studies demonstrating overlap between schizophrenia liability and other psychiatric disorders have generally been undertaken in samples that are not typical of disorders at the population level. For example, they are often enriched for severity, chronicity and attendance at specialist clinics. It is therefore important to determine whether findings from atypical samples generalise to samples ascertained to be representative of their source population. We employ two such samples in our study (New Zealand and UK) Previous studies employing the Avon Longitudinal Study of Parents and Children (ALSPAC), ${ }^{9,10}$ a birth cohort study based on children born in South West England (Avon; 1991-1992), demonstrate that schizophrenia PRSs were not associated with positive schizophrenia or depressive symptoms in adolescence (12-18 years), but was associated with negative symptoms and anxiety disorders. ${ }^{11}$ Hence, negative symptoms and anxiety may be more relevant to the transition to schizophrenia during adolescence than positive symptoms. Schizophrenia is frequently preceded by, and can be comorbid with, anxiety disorders; ${ }^{12}$ one reason for this is shared genetic aetiology. However, the ALSPAC study does not yet extend into adulthood and therefore it is unclear whether the pleiotropic effects of schizophrenia risk alleles on anxiety in the general population are transient, or whether they continue into adulthood. To clarify this, we used the Christchurch Health and Development Study (CHDS). The CHDS includes measures of mental health at multiple time points in adulthood; ${ }^{13-15}$ as these include multiple anxiety disorders (specific phobia, social phobia, panic disorders and generalised anxiety disorder [GAD]), we also investigated whether schizophrenia liability is increased across anxiety disorders or whether it shows any specificity to a particular diagnosis. We used the CHDS and ALSPAC data sets to investigate the populationlevel relationship between schizophrenia PRS and other forms of psychopathology: schizophrenia/schizophreniform disorder, manic/hypomanic episode, depression and alcohol dependence.

\section{Methods}

\section{Sample}

We employ two samples in our study. Our primary sample is the CHDS, which is an ongoing birth cohort of 1265 children born in 
the Christchurch (New Zealand) urban region in mid-1977. Participants have been studied on a total of 23 occasions from birth to age 35 years. ${ }^{13,14}$ The present analysis is based on data collected during assessments on the cohort at ages 21, 25, 30 and 35 years. Sample retention rates have remained high throughout the course of the study and, at age 35, the study was still able to assess $79 \%$ of the surviving cohort. Participant consent was obtained for all forms of data collection and all phases of the study have been subject to ethical approval. Ethical approvals relevant to the current research are from the Southern Health and Disability Ethics Committee (ref CTB/04/11/234/AM09, 16/STH/188/AM01).

We also employed the ALSPAC (www.bris.ac.uk/alspac/), which is a birth cohort study based on children born in South West England (Avon) between 1 April 1991 and 31 December 1992.9 Participants were not randomly selected, but the demographic characteristics of ALSPAC have been shown to be representative of the UK as a whole. ${ }^{9}$ The resource comprises a wide range of phenotypic and environmental measures in addition to biological samples, genetic (DNA on 11343 children, genomewide data on 8365 children and complete genome sequencing on 2000 children) and epigenetic (methylation sampling on 1000 children) information, and linkage to health and administrative records. The study website contains details of all the data, searchable through the data dictionary (www.bris.ac.uk/alspac/researchers/ data-access/data-dictionary/). This study received ethical approval from the ALSPAC Law and Ethics Committee and Local Research Ethics Committees (http://www.bristol.ac.uk/alspac/researchers/ research-ethics/).

\section{DNA extraction and genotyping}

In the CHDS sample, individuals between the ages of 28-30 years provided a DNA sample for genetic analysis. In $91.4 \%$ of cases, DNA was extracted from whole blood; for the remaining participants (8.6\%), saliva was collected using Oragene Collection Kits (DNA Genotek, Ottawa, Canada) and DNA was extracted according to the supplier's instructions. DNA extractions were completed at the Gene Structure and Function Laboratory, based at the University of Otago, Christchurch, New Zealand. ${ }^{16}$ Available samples were genotyped using Illumina Human660W-Quad v1 DNA Analysis BeadChips at the Mayo Clinic. ${ }^{15}$

Full details of sample and variant quality control are given in Supplementary Text and Supplementary Table 1 available at https://doi.org/10.1192/bjp.2018.227. Genotypes were imputed using the 1000 Genomes Phase 3 data set as a reference panel (see Supplementary Text). Principal component analysis was performed to produce population principal components for use as covariates in further analyses. The data set from which the risk alleles are derived are overwhelmingly of European ancestry so to maximise power and avoid confounding, ${ }^{17}$ analyses were limited to individuals of European ancestry as defined by genomic similarity to the European reference samples represented in the 1000 Genomes data set. ${ }^{18}$

\section{PRS construction}

The PRS is a summary score of the number of risk alleles carried by an individual, weighted according to their effect sizes, where risk allele and effect size are determined from an independent genomewide association study (GWAS) of the disorder (the 'discovery set'). ${ }^{3}$ PRSs can predict schizophrenia status in case-control studies and capture sufficient risk to examine genetic relationships between different diagnostic categories. ${ }^{5}$

PRSs were derived using the method employed by the International Schizophrenia Consortium. ${ }^{3}$ Risk alleles and odds ratios were taken from the discovery data set made available by the Schizophrenia Working Group of the Psychiatric Genomics
Consortium (PGC) which contained 35476 cases of the disorder and 46839 controls. ${ }^{5}$ To allow for the effects of linkage disequilibrium, variants were restricted to autosomal single-nucleotide polymorphisms (SNPs) in relative linkage equilibrium (using -clump in PLINK version 1.07 on a UNIX system ${ }^{19}$ with the PGC2 schizophrenia meta-analysis $P$-values, with a maximum $r^{2}$ of 0.25 and a window size of $500 \mathrm{~kb}$ ), leaving 87713 SNPs. PRSs were calculated for each CHDS participant as the mean number of risk alleles surpassing a particular association $P$-value threshold $\left(P_{\mathrm{T}}\right)$ in the PGC study, each weighted by its log-odds ratio, using the program PLINK. ${ }^{19}$ Primary analyses are based on scores constructed using a significance threshold $\left(P_{\mathrm{T}}\right)$ of 0.05 in the discovery GWAS as this is the modal threshold that captures the maximal variance to the schizophrenia phenotype in each of the subsamples of that meta-analysis. ${ }^{5}$ The risk scores were divided into quartiles for further analysis. For phenotypes that showed significant relationships with schizophrenia PRSs, secondary analyses were performed across a range of $P_{\mathrm{T}}$ values $\left(P_{\mathrm{T}}=1,0.5,0.3,0.2,0.1,0.01,0.001,1 \times\right.$ $10^{-4}, 1 \times 10^{-6}, 5 \times 10^{-8}$ ) to ensure the pattern of results was not particularly sensitive to the chosen threshold. Secondary analyses were also performed on these phenotypes by using continuous PRS values to ensure that our results were not sensitive to our use of PRS quartiles as a primary measure.

\section{Mental health outcomes (18-35 years)}

At ages 21, 25, 30 and 35 years, participants were administered a comprehensive mental health interview that assessed aspects of the individual's mental health and psychosocial adjustment over the period since the previous assessment. As part of this interview, the symptoms obtained were used to assign DSM-IV $(1994)^{20}$ mood disorders (major depression, manic/hypomanic episode); anxiety disorders (GAD, panic disorders, agoraphobia, social phobia, specific phobia) and alcohol dependence. Questioning was based on the relevant sections of the Composite International Diagnostic Interview (CIDI), ${ }^{21}$ which is a structured interview for mental/psychiatric disorders. The CIDI has been used extensively in community and epidemiological surveys of disorder. ${ }^{20}$ From age 30 years, interviews were extended to include symptoms required to make DSM-IV diagnoses of schizophrenia/schizophreniform disorder. Interviews were administered by trained lay interviewers, as per the design of the CIDI. In the majority of cases (approximately 80\%), interviews were conducted face to face or, if this was not possible (e.g. the participant was resident overseas), via telephone or Skype. Using these data, participants were first classified as to whether they met criteria for each of the above diagnoses in each of the interview periods from age 18-21, $21-25,25-30$ and $30-35$ years. This information was then combined over interview periods to classify participants as to whether they had ever met criteria for the DSM-IV diagnoses over the whole period of age 18-35 years. The diagnoses included in the present analysis and associated adult lifetime (18-35 years) prevalence estimates were as follows: major depression (47.6\%), manic or hypomanic episode (7.6\%), GAD (9.1\%), panic disorder with or without agoraphobia (14.2\%), social phobia (15.4\%), specific phobia (20.7\%), alcohol dependence (12.9\%) and schizophrenia/ schizophreniform disorder (2.0\%). The total number of anxiety disorders (defined as specific phobia, social phobia, panic disorder and GAD) was also calculated and used as a phenotypic variable. In this longitudinal study of lifetime adult disorder (up to age 30), consistent with aetiological pleiotropy, there was substantial comorbidity between domains of mood disorder (e.g., major depressive disorder, manic/hypomanic episode), anxiety, alcohol dependence and schizophrenia (odds ratios up to 30 depending on disorder; see Supplementary Table 2). 


\section{Sample size and statistical analysis}

The present analysis was based on a sample of 590 participants (286 male and 304 female) from CHDS who were observed on mental health outcomes from age 18 to 35 years, who were successfully genotyped on the Illumina chip and therefore for whom schizophrenia PRSs could be constructed. As above, analyses were limited to participants who were of European ancestry. Comparison of those included in the analysis with those not included showed no statistically significant differences in the observed rates of disorder (see Supplementary Text and Supplementary Table 3).

We performed regression of the phenotype variables on quartiles of schizophrenia PRSs (logistic regression for dichotomous outcomes, linear regression for the number of anxiety disorders). Gender and the first two population principal components were included in the regression as covariates. Analyses were conducted using the $\operatorname{glm}()$ function in $\mathrm{R}^{22}$ As phenotype measures were correlated, Bonferroni multiple testing correction would be overly conservative and so we used false discovery rate (FDR) multiple testing correction (in R) instead. ${ }^{23}$ Where significant associations were found between schizophrenia PRS and anxiety disorders, conditional analysis was conducted to determine whether these associations were independent of each other.

To examine the effect of comorbidity among anxiety disorders, we also performed a secondary association analysis comparing individuals with any one anxiety disorder with those with no anxiety disorders, and another comparing individuals with two or more anxiety disorders with those with no anxiety disorders (see Supplementary Table 2).

\section{Replication sample and analysis}

To replicate our novel finding from the CHDS study, we tested the relationship between schizophrenia PRS and mania/hypomania episode in the ALSPAC sample. The initial cohort contained 15445 participants with extensive baseline information from the first trimester of pregnancy onwards. Data were collected regularly at defined time intervals and is ongoing.

Hypomanic features in ALSPAC was assessed using the Hypomania Checklist (HCL-32) at age 22-23 years. The HCL-32 is a self-rating questionnaire designed to capture a lifetime history of hypomanic symptoms. ${ }^{24-27}$ It has been used extensively in clinical and non-clinical settings and is validated as a screening tool for bipolar disorder type II. Following a Rasch analysis for unidimensionality of the HCL-32, four items were identified as redundant and could be excluded. ${ }^{28}$ Our primary analysis in ALSPAC was based on the preferred definition of hypomanic episode in ALSPAC, which is defined as meeting a threshold score on the HCL-32 of 14 or more (out of 28), having symptoms for at least 2 days and a response of either a negative or both negative and positive impact of periods of elevated mood on family, social, or work life or on leisure. However, we tested a more stringent definition requiring a 4 day duration, as in CHDS.

Genetic data from 9912 participants were obtained using a genome-wide SNP genotyping platform (HumanHap550-Quad; Illumina). Following quality control, imputation and restriction to 1 young person per family, genetic data were available on 8230 individuals, of whom 2655 individuals were of European ancestry and had HCL-32 data. Based on the 2-day duration criterion, the number of individuals classed as having a hypomanic episode $(n=239)$ was comparable to that in CHDS $(\sim 7 \%)$, but this fell to $50(1.88 \%)$ when the 4 -day criterion was applied.

The schizophrenia PRS was constructed in the same way as for the CHDS sample (see above), using the PGC2 schizophrenia study as the discovery data set ${ }^{5}$ and a $P_{\mathrm{T}}$ threshold of 0.05 . The relationship between schizophrenia PRS and hypomanic episode was examined using logistic regression in STATA (v14.1 SE, Macintosh platform), including gender as a covariate. In both the CHDS and ALSPAC samples, schizophrenia PRSs were calculated blind to phenotype classification.

\section{Results}

Results for the CHDS sample are given in Table 1. The proportion of cases affected by each dichotomous phenotype within each quartile of schizophrenia PRSs are given in Fig. 1. Despite the very small number of individuals $(n=12)$ meeting criteria for schizophrenia/ schizophreniform disorder, higher schizophrenia PRS was associated with this phenotype, although evidence for this was weaker after correction for multiple testing (FDR-corrected $P=0.052$ ). Across anxiety disorders, schizophrenia PRS was associated both with GAD (FDR-corrected $P=0.018$ ) and panic disorder (FDR-corrected $P=0.034$ ) but not with specific or social phobias. When the association between schizophrenia PRS and GAD was conditioned on panic disorder, the result was no longer significant (FDR-corrected $P=0.086$ ). The same was true when the association between schizophrenia PRS and panic disorder was conditioned on GAD (FDR-corrected $P=0.22$ ). Among the other phenotypes, higher schizophrenia PRS was associated with manic/hypomanic episode (FDR-corrected $P=0.021$ ), but not with depression or alcohol dependence. Schizophrenia PRS was also associated with number of anxiety disorders (FDR-corrected $P=0.018$; Fig. 2). When we performed separate association analyses on cases with a single anxiety disorder and those with two or more, the latter were much more significantly associated with schizophrenia PRS (Supplementary Table 4).

All phenotypes showing significant associations with schizophrenia PRSs in our primary analysis $\left(P_{\mathrm{T}}=0.05\right)$ also showed evidence for association across multiple thresholds, which shows our results were not highly sensitive to our choice of this threshold (see Supplementary Table 5). In some instances, the associations were much more significant at an alternative threshold. For example, for total number of anxiety disorders the strongest evidence for association $\left(P=8.1 \times 10^{-6}\right)$ was observed at $P_{\mathrm{T}}=0.01$.

Similarly, when we used continuous PRSs instead of PRS quartiles as a secondary analysis, all phenotypes showing significant associations with schizophrenia PRS remained significantly associated, which shows our results are not sensitive to our decision to use PRS quartiles (see Supplementary Table 6).

Finally, we confirmed our observation of association between manic/hypomanic episode and schizophrenia PRS in ALSPAC $(P$ of 0.002 for symptom duration criterion of 2 days, $P$ of 0.005 for symptom duration criterion of 4 days).

\section{Discussion}

This study investigated the relationship between schizophrenia genetic liability and psychiatric diagnoses in early adulthood in a New Zealand population cohort. We found associations between schizophrenia PRS and manic/hypomanic episodes and anxiety disorders, but other phenotypes were either not significantly associated, or, in the case of schizophrenia/schizophreniform disorder, did not survive correction for multiple testing.

\section{Schizophrenia PRS and schizophrenia}

An initial association between schizophrenia PRS and schizophrenia/schizophreniform disorder did not remain significant following correction for multiple testing (Table 1). These inconclusive results are consistent with previous population studies in adolescents 


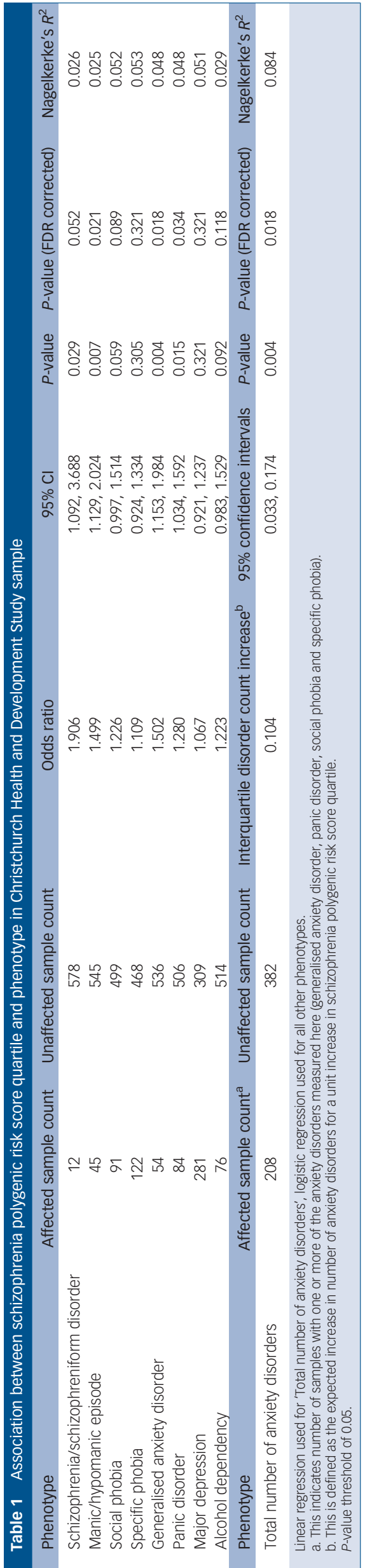

which found no evidence of association between schizophrenia PRS and psychotic experiences. The symptoms included in assigning schizophrenia/schizophreniform diagnoses here included both negative and positive symptoms, which may explain the weak evidence for association, as a previous study (based on ALSPAC) found an association between PRS and negative symptoms but not positive symptoms. ${ }^{11}$

The low frequency of schizophrenia/schizophreniform diagnoses in our sample (2.0\%) compared with GAD (9.2\%) and manic/ hypomanic episode $(7.6 \%)$ is a likely reason for the weak evidence for association observed here. Consistent with this and our expectations, it is notable that the point estimate for the effect size for association (odds ratio of 1.906) was stronger for schizophrenia/ schizophreniform than for any other disorder. In addition to a genuine relative rarity of schizophrenia/schizophreniform in the population compared with other diagnoses, we also note the symptoms required to assign that diagnosis were only obtained at two time points in the CHDS study (30-35 years), whereas those required to assign other diagnoses were measured across four time points. Thus, it is possible we have missed a higher fraction of true schizophrenia/schizophreniform diagnoses than may be the case for other diagnoses.

\section{Schizophrenia PRS and anxiety disorders}

We found strong evidence of association between schizophrenia PRS and GAD, and schizophrenia PRS and panic disorder (Table 1). A higher schizophrenia PRS was also strongly associated with total number of anxiety disorders.

Anxiety disorders in adolescence were associated with schizophrenia PRS in the ALSPAC data set, suggesting that anxiety may be a prodromal feature in schizophrenia. ${ }^{11}$ Similarly, the associations between schizophrenia PRS, GAD and panic disorder show that the relationship between schizophrenia genetic risk and anxiety persists into adulthood. Whether this relationship reflects direct causal overlap between the disorders or pleiotropic effects of schizophrenia-associated genes on anxiety disorder is unclear. As a next step, it may be interesting to examine whether this genetic overlap is driven by genes belonging to specific biological pathways, particularly those with some evidence for involvement in schizophrenia.

The conditional analysis suggested that the GAD result was not conditionally independent of panic disorder and vice versa. Alongside the relationship between schizophrenia PRS and total number of anxiety disorders, this suggests that as anxiety disorder comorbidity increases, so does the relevance of schizophrenia genetic risk. This is further emphasised by the secondary analysis showing that almost all of the association signal for schizophrenia PRS comes from cases with two or more anxiety disorders (Supplementary Table 4).

\section{Schizophrenia PRS and other psychopathological variables}

We also found a positive association between schizophrenia PRS and manic/hypomanic episode in the CHDS sample. This positive association was replicated in the ALSPAC population study, suggesting that it is unlikely to be due to chance. The links between schizophrenia and bipolar disorder are well documented at both the phenotypic and genetic levels, with many individuals with schizophrenia showing manic symptoms and many individuals with bipolar disorder displaying psychotic symptoms. However, no previous population study has shown evidence of a relationship between schizophrenia genetic risk and manic/hypomanic episode. Thus this finding is a novel demonstration of genetic overlap between the two disorders across the adult population beyond clinical populations. 


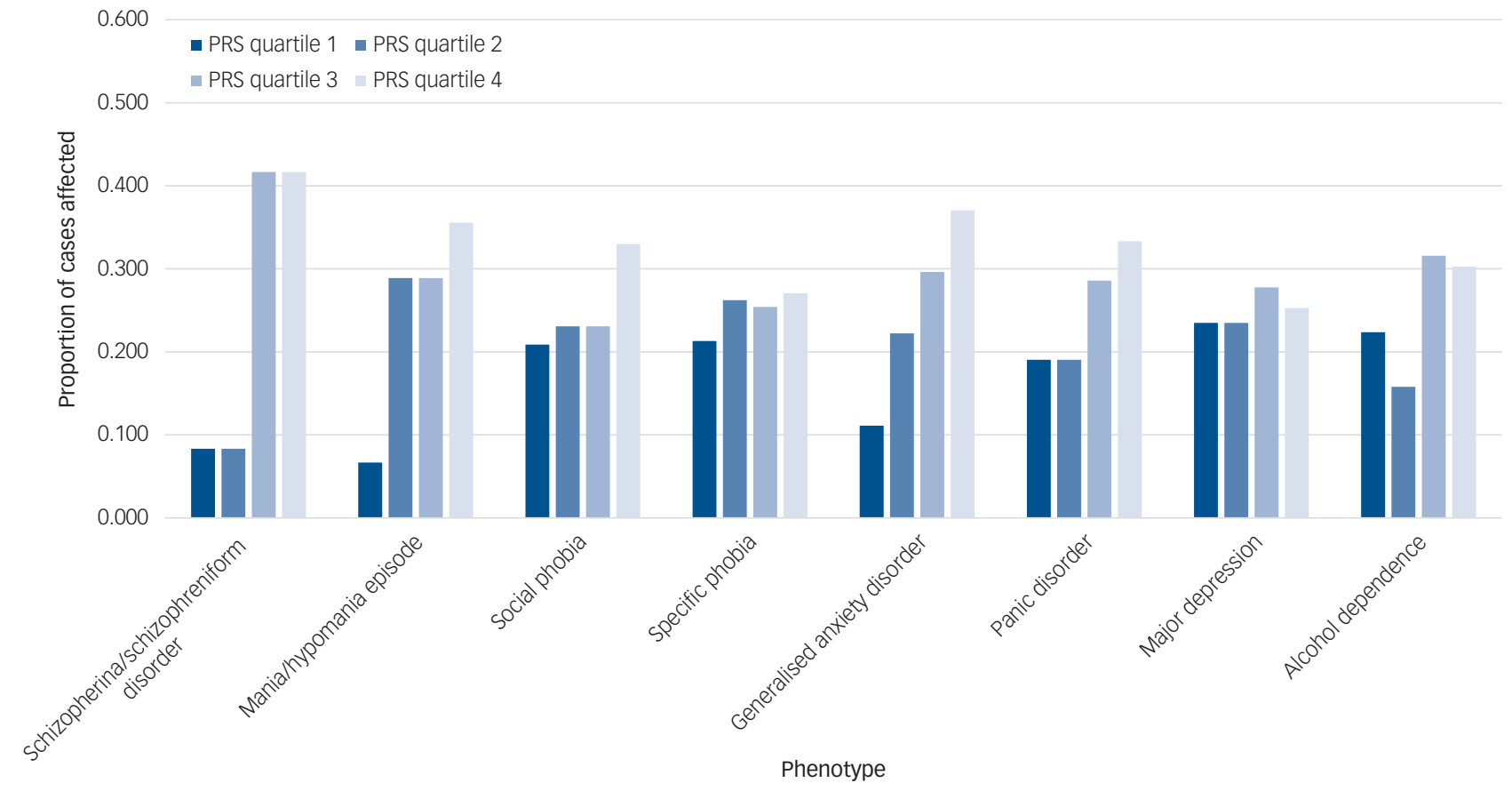

Fig. 1 Proportion of cases affected at schizophrenia polygenic risk score quartiles 1-4 for each phenotype in the Christchurch Health and Development Study (CHDS). PRS, polygenic risk score.

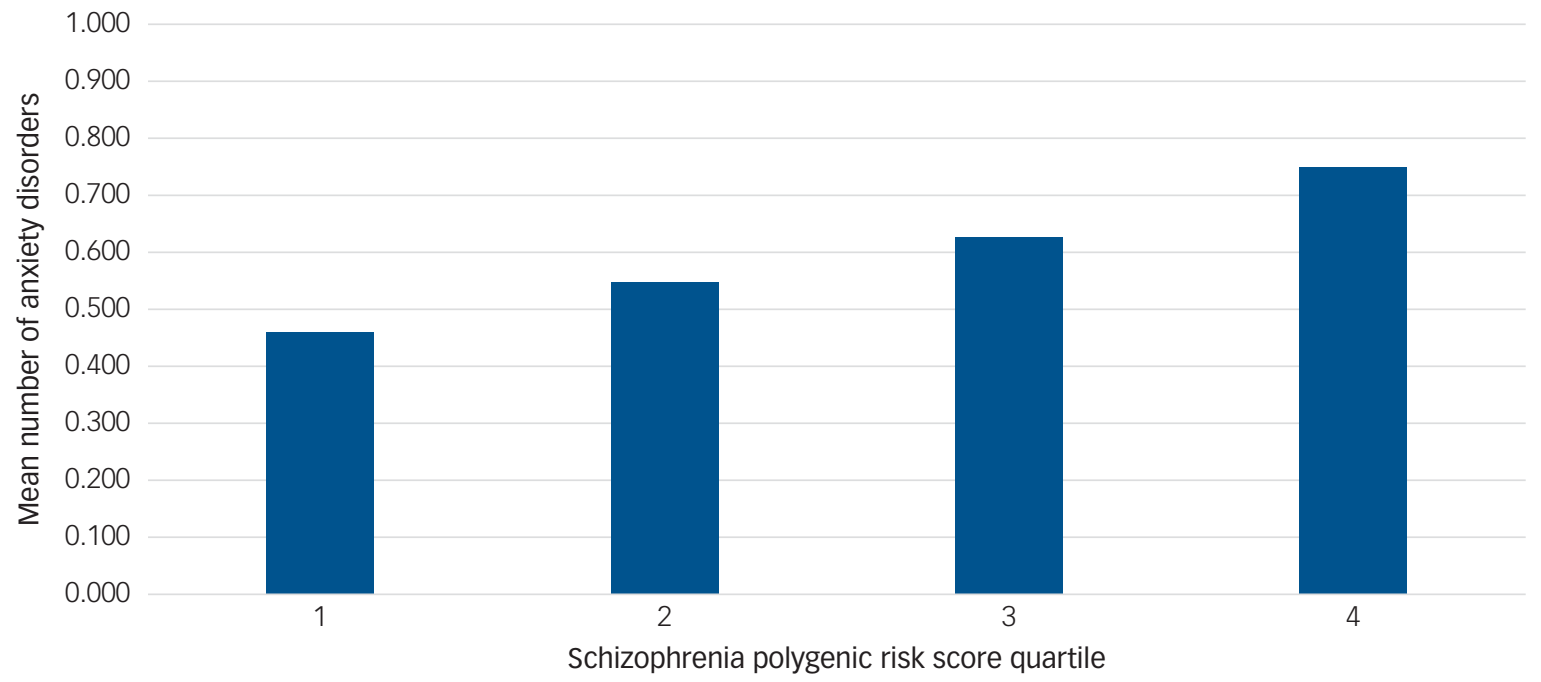

Fig. 2 Mean number of anxiety disorders per sample at schizophrenia polygenic risk score (SZ PRS) quartile 1-4 in the Christchurch Health and Development Study (CHDS) sample.

Although anxiety and manic/hypomanic episode may be affected by schizophrenia genetic risk, it is unclear the extent to which they show a specific relationship with schizophrenia. Anxiety is a common symptom in a wide variety of psychiatric disorders, whereas mania and hypomania are the key features of bipolar disorder. The extent to which these symptoms and schizophrenia genetic risk index risk for a range of psychiatric disorders including schizophrenia, is not yet fully understood. It is also unclear how these phenotypes and schizophrenia PRS affect specific symptoms or cognitive deficits. However, work with PRSs and linkage disequilibrium (LD) score techniques shows that there is considerable genetic overlap between different psychiatric disorders. ${ }^{29}$

\section{Strengths and limitations}

This study has limitations besides those discussed above. The CHDS sample size is not as large as the ALSPAC or other relevant studies, so the sample may be insufficiently powered to detect true associations of small effect size. Generalisability of findings should also be considered relative to the sample attributes of both the New Zealand and UK cohorts included in the present study. The CHDS analysis 
presented here is based on European-descent samples from New Zealand, and so results may not generalise to other data sets from countries with different healthcare systems and cultural features. However, the presence of similar results in the ALSPAC cohort from the UK mitigates this issue considerably. As with all longitudinal cohort studies, there is also the possibility of different levels of attrition between groups of samples. A greater chance of non-response or missing data due to psychiatric symptoms for samples with high schizophrenia PRS would reduce power to find an association between the PRS and those symptoms.

One of the strengths of the study is that the PRS discovery set, the PGC2 meta-analysis ( $n=82315$ ), is the largest publicly available, which should minimise measurement error in the scores and hence improve the power of studies using these scores for analyses in target samples. ${ }^{5}$ The CHDS sample is also a relatively richly phenotyped sample, which enables us to examine effects on specific anxiety disorders rather than only anxiety disorders as a whole.

\section{Impact of this study and future directions}

This study shows a positive association between schizophrenia PRS and anxiety in adulthood where previous associations covered adolescence, suggesting that schizophrenia genetic risk can have an impact on anxiety aetiology in the absence of a clinical diagnosis of schizophrenia. It also shows greater specificity than previous work, which only found association between the presence of any anxiety disorder and schizophrenia PRS. Although all anxiety disorders considered here make some contribution to the association, the strongest drivers of this effect are GAD and panic disorder. We also showed association between schizophrenia PRS and manic/hypomanic episodes in a population cohort, emphasising the overlap between these two disorders. Further work in larger population cohorts with measures of adult psychiatric dysfunction would be useful to replicate these results.

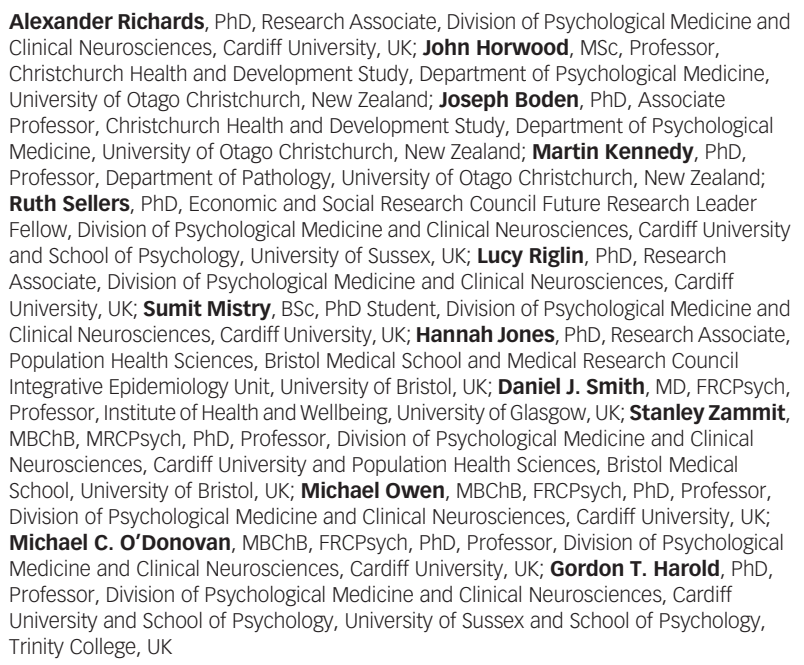

First received 22 Dec 2017, final revision 16 Jul 2018, accepted 18 Sep 2018

\section{Supplementary material}

Supplementary material is available online at https://doi.org/10.1192/bjp.2018.227.

\section{Funding}

The Christchurch Health and Development Study has been supported by funding from the Health Research Council of New Zealand, the National Child Health Research Foundation (Cure Kids), the Canterbury Medical Research Foundation, the New Zealand Lottery Grants Board, the University of Otago, the Carney Centre for Pharmacogenomics, the James Hume Bequest Fund, US National Institutes of Health grant MHO77874 and National Institute on Drug Abuse grant R01DA024413. G.T.H. was supported by an Economic and Social Research Council project grant award (ES/L014718/1). R.S. is supported by an Economic and Social Research Council project grant award (ES/N003098/1). Cardiff University researchers were supported by the Medical Research Council Centre (G0800509) and Programme Grant (G0801418). The UK Medical Research Council and the Wellcome Trust (102215/2/13/2) as well as the University of Bristol provide core support for the Avon Longitudinal Study of Parents and Children. Genome-wide association study data were generated by Sample Logistics and Genotyping Facilities at the Wellcome Trust Sanger Institute and LabCorp (Laboratory Corporation of America) using support from 23 andMe. DJ.S. is supported by a Lister Institute Prize Fellowship (2016-2021). H.J. and S.Z. are supported by the National Institute for Health Research Bristol Biomedical Research Centre.

\section{Acknowledgements}

We thank all families who took part in the Avon Longitudinal Study of Parents and Children (ALSPAC), the midwives for their help in recruiting them and the whole ALSPAC team, which includes interviewers, computer and laboratory technicians, clerical workers, research scientists, volunteers, managers, receptionists and nurses.

\section{References}

1 Gottesman II, Shields J. A polygenic theory of schizophrenia. Proc Natl Acad SCi US A 1967; 58: 199-205.

2 Cardno AG, Gottesman II. Twin studies of schizophrenia: from bow-and-arrow concordances to star wars Mx and functional genomics. Am J Med Genet 2000; 97: 12-7.

3 The International Schizophrenia Consortium. Common polygenic variation contributes to risk of schizophrenia and bipolar disorder. Nature 2009; 460 : 748-52.

4 Sullivan PF, Daly MJ, O'Donovan M. Genetic architectures of psychiatric disorders: the emerging picture and its implications. Nat Rev Genet 2012; 13: 537-51.

5 Schizophrenia Working Group of the Psychiatric Genomics Consortium Biological insights from 108 schizophrenia-associated genetic loci. Nature 2014; 511: 421-7.

6 Whalley HC, Adams MJ, Hall LS, Clarke TK, Fernandez-Pujals AM, Gibson J, et al. Dissection of major depressive disorder using polygenic risk scores for schizophrenia in two independent cohorts. Transl. Psychiatry 2016; 6: e938.

7 Hubbard L, Tansey KE, Rai D, Jones P, Ripke S, Chambert KD, et al. Evidence of common genetic overlap between schizophrenia and cognition. Schizophr Bull 2016; 42: 832-42.

8 Riglin L, Collishaw S, Richards A, Thapar AK, Maughan B, O'Donovan MC, et al. Schizophrenia risk alleles and neurodevelopmental outcomes in childhood: a population-based cohort study. Lancet Psychiatry 2017; 4: 57-62.

9 Boyd A, Golding J, Macleod J, Lawlor DA, Fraser A, Henderson J, et al. Cohort

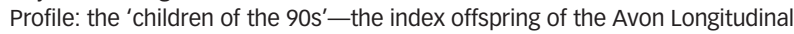
Study of Parents and Children. Int J Epidemiol 2013; 42: 111-27.

10 Fraser A, Macdonald-Wallis C, Tilling K, Boyd A, Golding J, Davey Smith G, et al. Cohort Profile: the Avon Longitudinal Study of Parents and Children: ALSPAC mothers cohort. Int J Epidemiol 2013; 42: 97-110.

11 Jones HJ, Stergiakouli E, Tansey KE, Hubbard L, Heron J, Cannon M, et al. Phenotypic manifestation of genetic risk for schizophrenia during adolescence in the general population. JAMA Psychiat 2016; 73: 221-8.

12 Buckley PF, Miller BJ, Lehrer DS, Castle DJ. Psychiatric comorbidities and schizophrenia. Schizophr Bull 2009; 35: 383-402.

13 Fergusson DM, Horwood $\mathrm{L}$. The christchurch health and development study. In The Christchurch Experience: 40 Years of Research and Teaching (eds P Joyce, G Nicholls, K Thomas, T Wilkinson): 79-87. University of Otago, 2013.

14 Fergusson DM, Horwood LJ. The Christchurch Health and Development Study: review of findings on child and adolescent mental health. Aust N Z J Psychiatry 2001; 35: 287-96.

15 Adkins DE, Clark SL, Copeland WE, Kennedy M, Conway K, Angold A, et al. Genome-wide meta-analysis of longitudinal alcohol consumption across youth and early adulthood. Twin Res Hum Genet 2015; 18: 335-47.

16 Fergusson DM, Horwood LJ, Miller A, Kennedy MA. Life stress, 5-HTTLPR and mental disorder: Findings from a 30 year longitudinal study. Br J Psychiatry 2011; 198: 129-35. 
17 Martin AR, Gignoux CR, Walters RK, Wojcik GL, Neale BM, Gravel S, et al. Human demographic history impacts genetic risk prediction across diverse populations. Am J Hum Genet 2017; 100: 635-49.

18 The 1000 Genomes Project Consortium. A global reference for human genetic variation. Nature 2015; 526: 68-74.

19 Purcell S, Neale B, Todd-Brown K, Thomas L, Ferreira MA, Bender D, et al. PLINK: a tool set for whole-genome association and population-based linkage analyses. Am J Hum Genet 2007; 81: 559-75.

20 American Psychiatric Association. Diagnostic and Statistical Manual of Mental Disorders: DSM-IV. American Psychiatric Association, 1994.

21 World Health Organization. Composite International Diagnostic Interview (CIDI). World Health Organization, 1993.

22 RC Team. R: A Language and Environment for Statistical Computing. R Foundation for Statistical Computing, 2014

23 Benjamini $Y$, Hochberg Y. Controlling the false discovery rate: a practical and powerful approach to multiple testing. J Roy Stat SOC B Met 1995; 57: 289-300.

24 Angst J, Azorin J-M, Bowden CL, Perugi G, Vieta E, Gamma A, et al. Prevalence and characteristics of undiagnosed bipolar disorders in patients with a major depressive episode the BRIDGE study. Arch Gen Psychiatry 2011; 68: 791-9.
25 Meyer TD, Schrader J, Ridley M, Lex C. The Hypomania Checklist (HCL) Systematic review of its properties to screen for bipolar disorders. Compr Psychiatry 2014; 55: 1310-21.

26 Forty L, Smith D, Jones L, Jones I, Caesar S, Fraser C, et al. Identifying hypomanic features in major depressive disorder using the hypomania checklist (HCL-32). J Affect Disord 2009; 114: 68-73.

27 Carta MG, Hardoy MC, Cadeddu M, Murru A, Campus A, Morosini PL, et al. The accuracy of the Italian version of the Hypomania Checklist (HCL-32) for the screening of bipolar disorders and comparison with the Mood Disorde Questionnaire (MDQ) in a clinical sample. Clin Pract Epidemol Ment Health 2006; 2: 2.

28 Court H, Forty L, Jones L, Gordon-Smith K, Jones I, Craddock N, et al. Improving the psychometric utility of the hypomania checklist (HCL-32): a Rasch analysis approach. J Affect Disord 2014: 152-4, 448-53.

29 Cross-Disorder Group of the Psychiatric Genomics Consortium. Identification of risk loci with shared effects on five major psychiatric disorders: a genomewide analysis. Lancet 2013; 381: 1371-9.

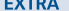

\section{psychiatry in history}

\section{Sir Thomas Browne: Boulimia Centenaria}

\section{Greg Wilkinson}

Sir Thomas Browne (1605-1682), a polymath, practised medicine in Norwich. He communicated the case report below, which was read by his son to the Royal Society on 1 February 1672 . Sir Thomas supposed that the cause was sal esurinum: the 'hungry salt', regarded as promoting gastric digestion and appetite by van Helmont in 1624

There is a woman now living in Yarmouth named Elizabeth Michell, an hundred and two yeares old; a person of 4 feet and an half high, very leane, very poore, and living in a mean roome, with pitifull accomodation. She had a sonne after she was past fifty. Though she answers well enough unto ordinary questions, yet she apprehends her eldest daughter to be her mother, but what is most remarkable concerning her is a kinde of Boulimia or dogge appetite. She greedily eating day and night whatever allowance friends or charitable persons afford her, drinking beere or water, and making little distinction or refusal of any food, either of broathes, flesh, fish, apples, peares, and any coarse foode which shee eateth in no small quantity, in so much that the overseers for the poore have of late been forced to augment her weekely allowance. Shee sleeps indifferently well till hunger awakes her, then she must have no ordinary supply whither in the day or night. She vomits not, nor is very laxative This is the oldest example of the Sal esurinum chymicorum which I have taken notice of. Though I am ready to afford my charity unto her yet I should be loath to spend a peece of Ambregris I have upon her and to afford her 6 grains to every dosis, till I founde some effect in moderating her appetite, though $\mathrm{y}^{t}$ be esteemed a great specifick in her condition. (Royal Society Archives: CL.P/14i/14.)

Copyright @ The Royal College of Psychiatrists 2019 